\title{
DENSITY ESTIMATES \\ FOR A NONLOCAL VARIATIONAL MODEL VIA THE SOBOLEV INEQUALITY
}

\author{
OVIDIU SAVIN AND ENRICO VALDINOCI
}

Abstract. We consider the minimizers of the energy

$$
\|u\|_{H^{s}(\Omega)}^{2}+\int_{\Omega} W(u) d x
$$

with $s \in(0,1 / 2)$, where $\|u\|_{H^{s}(\Omega)}$ denotes the total contribution from $\Omega$ in the $H^{s}$ norm of $u$, and $W$ is a double-well potential. By using a fractional Sobolev inequality, we give a new proof of the fact that the sublevel sets of a minimizer $u$ in a large ball $B_{R}$ occupy a volume comparable with the volume of $B_{R}$.

Given $s \in(0,1 / 2)$ and $\Omega \subseteq \mathbb{R}^{n}$, with $n \geqslant 2$, we define

$$
\mathscr{K}(u ; \Omega):=\frac{1}{2} \int_{\Omega} \int_{\Omega} \frac{|u(x)-u(y)|^{2}}{|x-y|^{n+2 s}} d x d y+\int_{\Omega} \int_{\mathscr{C} \Omega} \frac{|u(x)-u(y)|^{2}}{|x-y|^{n+2 s}} d x d y .
$$

We take $W$ to be a double-well potential, more precisely, we assume that $W$ : $[-1,1] \rightarrow[0, \infty)$

$$
\begin{gathered}
W \in C^{2}([-1,1]), \quad W( \pm 1)=0, \quad W>0 \quad \text { in }(-1,1) \\
W^{\prime}( \pm 1)=0, \quad \text { and } \quad W^{\prime \prime}( \pm 1)>0 .
\end{gathered}
$$

The energy functional we are interested in is the sum of the nonlocal contribution given by $\mathscr{K}$ and a local one induced by $W$, i.e., we define

$$
\mathscr{E}(u ; \Omega):=\mathscr{K}(u ; \Omega)+\int_{\Omega} W(u(x)) d x .
$$

We say that $u$ is a minimizer in $\Omega$ if $\mathscr{E}(u ; \Omega)<\infty$ and

$$
\mathscr{E}(u ; \Omega) \leqslant \mathscr{E}(v ; \Omega)
$$

for any $v$ which coincides with $u$ in $\mathscr{C} \Omega$. It is easy to see that minimizers satisfy an Euler-Lagrange equation of nonlocal type, which is driven by an elliptic integral operator of fractional type. More precisely, a minimizer $u$ is a solution of

$$
(-\Delta)^{s} u(x)+W^{\prime}(u(x))=0,
$$

where $(-\Delta)^{s}$ is the fractional power of the positive operator $-\Delta$, up to a normalizing multiplicative constant, that we neglect. More explicitly,

$$
(-\Delta)^{s} u(x):=\int_{\mathbb{R}^{n}} \frac{u(x)-u(y)}{|x-y|^{n+2 s}} d y
$$

OS has been supported by NSG grant 0701037. EV has been supported by FIRB project "Analysis and Beyond" and GNAMPA project "Equazioni nonlineari su varietà: proprietà qualitative e classificazione delle soluzioni". Part of this work was carried out while EV was visiting Columbia University. We thank Guido de Philippis for an interesting discussion. 
and the integral is understood in the principal value sense.

From the physical point of view, the functional in (2) may be seen as a nonlocal extension of the classical Allen-Cahn model for phase coexistence (for the latter, see, e.g., [12]), and its interfaces may be related with suitable nonlocal minimal surfaces. Roughly speaking, the double-well potential $W$ tries to drive the minimizers towards the pure phases -1 and +1 ; on the other hand, up to scaling the space variables, the term $\mathscr{K}$ may be seen as a penalization which prevents the formation of unnecessary interfaces and makes the problem consistent from the mathematical point of view. The main difference between (2) and the classical Allen-Cahn model is that we have here a fully nonlocal interaction $\mathscr{K}$ instead of the usual "kinetic term" $\int_{\Omega}|\nabla u(x)|^{2} d x$.

From the mathematical point of view, the term $\mathscr{K}$ may also be regarded as the square of a (semi)norm in a fractional Sobolev space $H^{s}$ (say, $\|u\|_{H^{s}(\Omega)}^{2}$, see, e.g., [5] and the more comprehensive bibliography on this quoted there).

We refer to [2, 7, 8, for the precise definitions and some basic results on the nonlocal minimal surfaces linked with the limit interface of the functional in (2), and to [13, 14, for a more detailed discussion and motivation. For related nonlocal problem of phase segregation with physical importance, see also [1, 3, 10, 11. Moreover, we recall that the nonlocal contribution $\mathscr{K}$ may be seen as arising from a long range interaction of particles, in connection with some statistical mechanics model (see, e.g., 9]).

In 14, we have obtained, among others, the following result:

Theorem 1. Let $u$ be a minimizer of $\mathscr{E}$ in $B_{R}$. Then for any $\theta_{1}, \theta_{2} \in(-1,1)$ such that

$$
u(0)>\theta_{1},
$$

we have that

$$
\left|\left\{u>\theta_{2}\right\} \cap B_{R}\right| \geqslant \bar{c} R^{n}
$$

if $R \geqslant \bar{R}\left(\theta_{1}, \theta_{2}\right)$.

The constant $\bar{c}>0$ depends only on $n, s$ and $W$ and $\bar{R}\left(\theta_{1}, \theta_{2}\right)$ is a large constant that depends also on $\theta_{1}$ and $\theta_{2}$.

Theorem 1 follows in the streamline of the density estimates for sublevel sets of minimizers, whose study was started in [6] for the Allen-Cahn equation. Estimate (4) is quite meaningful in applications, since, from the physical viewpoint, it represents an estimate on the probability of finding a phase in a given portion of the medium. We refer to 14 for further references about related density estimates, and for further comments about the important consequence that these estimates have in the applications (such as geometric results on $\Gamma$-convergence, uniform convergence of rescaled interfaces, rigidity and symmetry properties, etc.).

Theorem 1 has been proved in 14 by using a fine estimate on a weighted double integral. The purpose of this note is to perform an alternative proof, by using a fractional Sobolev inequality.

Such an alternative proof is given below. Then, in the appendix, we give a simple, essentially self-contained, proof of the fractional Sobolev inequality in use.

The preliminary computations for the proof of Theorem 1 are in common with [14], but the rest of the proof given here is conceptually and technically quite different. Indeed, the proof given in 14 is more general (for instance, it works also for the 
case $s \in[1 / 2,1)$, in which the limit interface of the nonlocal problem is the classical, i.e. local, perimeter, and the technique used also plays an important role in the study of the $\Gamma$-convergence of the rescaled functional performed in [13]). On the other hand, the proof in [14] is somewhat more difficult, since it is based on an "ad hoc" fine measure theoretic result, namely Theorem 1.6 there, which, roughly speaking, estimates the energy for making the phase change based on the nonlocal integral contribution. In this paper, this will be achieved more directly, via a fractional Sobolev inequality, and this makes the argument technically simpler (and also closer in spirit to the proof of [6] for the classical Allen-Cahn model, where the standard Sobolev inequality was used).

Proof of Theorem 1. First of all, we observe that, by (1), there exists a small constant $c>0$ such that 1

$$
\begin{aligned}
& W(t) \geqslant W(r)+c(1+r)(t-r)+c(t-r)^{2} \text { when }-1 \leqslant r \leqslant t \leqslant-1+c \\
& \text { and } W(r)-W(t) \leqslant(1+r) / c \text { when }-1 \leqslant r \leqslant t \leqslant+1 .
\end{aligned}
$$

We fix $\theta_{\star}:=\min \left\{\theta_{1}, \theta_{2},-1+c\right\}$, with $c$ as in (5).

Now, we recall a useful barrier that was constructed in Lemma 3.1 in [14]:

Lemma 2. Given any $\tau>0$, there exists a constant $C>1$, possibly depending on $n, s$ and $\tau$, such that the following holds: for any $R \geqslant C$, there exists a rotationally symmetric function

$$
w \in C\left(\mathbb{R}^{n},\left[-1+C R^{-2 s}, 1\right]\right),
$$

with

$$
w=1 \text { in } \mathscr{C} B_{R},
$$

such that

$$
-(-\Delta)^{s} u(x)=\int_{\mathbb{R}^{n}} \frac{w(y)-w(x)}{|x-y|^{n+2 s}} d y \leqslant \tau(1+w(x))
$$

and

$$
\frac{1}{C}(R+1-|x|)^{-2 s} \leqslant 1+w(x) \leqslant C(R+1-|x|)^{-2 s}
$$

for any $x \in B_{R}$.

As in [14], we define

$$
A(R):=c \int_{B_{R} \cap\left\{w<u \leqslant \theta_{\star}\right\}}(u-w)^{2} d x \quad \text { and } \quad V(R):=\left|\left\{u>\theta_{\star}\right\} \cap B_{R}\right| .
$$

Also, we fix $K \geqslant 2\left(R_{o}+1\right)$, to be taken suitably large in the sequel (possibly in dependence of $\theta_{\star}$ ), where $R_{o}$ is also suitably large (at the end, roughly speaking, this will give $\bar{R}$ as in the statement of Theorem [1 up to constants), and $R>2 K$. We take $w$ to be the function constructed in Lemma 2 with $\tau:=c / 4$, and we define $v(x):=\min \{u(x), w(x)\}$.

\footnotetext{
${ }^{1}$ It may be worth to remark that, in fact, Theorem 1 is proven here simply under condition (5), which is weaker than (1).
} 
Then, from formula (3.34) of [14], one knows that

$$
A(R)+\mathscr{K}\left(u-v ; B_{R}\right)+\frac{c}{2} \int_{B_{R} \cap\left\{w<u \leqslant \theta_{\star}\right\}}(1+w)(u-w) d x
$$

$$
\leqslant C \int_{0}^{R}(R+1-t)^{-2 s} V^{\prime}(t) d t
$$

Now, we observe that $u-v=0$ outside $B_{R}$, and so the Sobolev-type inequality (see Theorem 7) gives that

$$
\begin{aligned}
& \mathscr{K}\left(u-v ; B_{R}\right)=\mathscr{K}\left(u-v, \mathbb{R}^{n}\right) \\
& \quad \geqslant c_{1}\|u-v\|_{L^{2 n /(n-2 s)}\left(\mathbb{R}^{n}\right)}^{2}=c_{1}\|u-v\|_{L^{2 n /(n-2 s)}\left(B_{R}\right)}^{2}
\end{aligned}
$$

for a suitable $c_{1}>0$.

Now, by (7),

$$
w<-1+\left(1+\theta_{\star}\right) / 2 \text { in } B_{R-K},
$$

as long as $K$ is large enough. Hence, we have that

$$
|u-v| \geqslant u-v \geqslant u-w \geqslant\left(1+\theta_{\star}\right) / 2 \quad \text { in } B_{R-K} \cap\left\{u>\theta_{\star}\right\}
$$

and so

$$
\begin{aligned}
\| u- & v \|_{L^{2 n /(n-2 s)}\left(B_{R}\right)}^{2} \geqslant\left(\int_{B_{R-K} \cap\left\{u>\theta_{\star}\right\}}|u-v|^{2 n /(n-2 s)}\right)^{(n-2 s) / n} \\
& \geqslant\left(\int_{B_{R-K} \cap\left\{u>\theta_{\star}\right\}}\left(\left(1+\theta_{\star}\right) / 2\right)^{2 n /(n-2 s)}\right)^{(n-2 s) / n}=c_{2} V(R-K)^{(n-2 s) / n},
\end{aligned}
$$

for a suitable $c_{2}>0$ (possibly depending on $\theta_{\star}$, hence on $\theta_{1}$ and $\theta_{2}$, which have been fixed at the beginning).

Then, recalling (8) and (9), we obtain that

$$
c_{3} V(R-K)^{(n-2 s) / n} \leqslant \int_{0}^{R}(R+1-t)^{-2 s} V^{\prime}(t) d t,
$$


for a suitable $c_{3}>0$. Now, we integrate (11) in $R \in[\rho,(3 / 2) \rho]$, with $\rho \geqslant 2 K$, and we use that $s \in(0,1 / 2)$ to obtain that

$$
\begin{aligned}
& \frac{c_{3}}{2} \rho V(\rho-K)^{(n-2 s) / n} \\
\leqslant & c_{3} \int_{\rho}^{(3 / 2) \rho} V(R-K)^{(n-2 s) / n} d R \\
\leqslant & \int_{\rho}^{(3 / 2) \rho}\left(\int_{0}^{R}(R+1-t)^{-2 s} V^{\prime}(t) d t\right) d R \\
\leqslant & \int_{0}^{(3 / 2) \rho}\left(\int_{t}^{(3 / 2) \rho}(R+1-t)^{-2 s} d R\right) V^{\prime}(t) d t \\
= & \frac{1}{1-2 s} \int_{0}^{(3 / 2) \rho}\left[((3 / 2) \rho+1-t)^{1-2 s}-1\right] V^{\prime}(t) d t \\
\leqslant & \frac{((3 / 2) \rho+1)^{1-2 s}}{1-2 s} \int_{0}^{(3 / 2) \rho} V^{\prime}(t) d t \\
\leqslant & \frac{4^{1-2 s}}{1-2 s} \rho^{1-2 s} V((3 / 2) \rho),
\end{aligned}
$$

that is, for any $\rho \geqslant 2 K$,

$$
\rho^{2 s} V(\rho-K)^{(n-2 s) / n} \leqslant \tilde{C} V((3 / 2) \rho),
$$

for a suitable $\tilde{C}>0$.

We take $r:=\rho-K$ in (12), and we obtain that

$$
r^{2 s} V(r)^{(n-2 s) / n} \leqslant C V(2 r)
$$

for a suitable $C \geqslant K^{2}$, as long as $r \geqslant C$ (notice that $C$ may depend on $K$, which is now fixed once and for all).

Now, we recall the following general, inductive result, for the proof of which we refer to Lemma 3.2 of [14]:

Lemma 3. Let $\sigma, \mu \in(0,+\infty), \nu \in(\sigma,+\infty)$ and $\gamma, R_{o}, C \in(1,+\infty)$.

Let $V:(0,+\infty) \rightarrow(0,+\infty)$ be a nondecreasing function. For any $r \in\left[R_{o},+\infty\right)$, let

Suppose that

$$
\alpha(r):=\min \left\{1, \frac{\log V(r)}{\log r}\right\} .
$$

and

$$
V\left(R_{o}\right) \geqslant \mu
$$

$$
r^{\sigma} \alpha(r) V(r)^{(\nu-\sigma) / \nu} \leqslant C V(\gamma r), \quad \text { for any } r \in\left[R_{o},+\infty\right) .
$$

Then, there exist $c \in(0,1)$ and $R_{\star} \in\left[R_{o},+\infty\right)$, possibly depending on $\mu, \nu, \gamma, R_{o}$ and $C$, such that

$$
V(r) \geqslant c r^{\nu}, \quad \text { for any } r \in\left[R_{\star},+\infty\right) .
$$

Then, we apply Lemma 3 with $\sigma:=2 s$ and $\gamma:=2$ and we deduce from (13) that $V(R) \geqslant c_{o} R^{n}$ for large $R$, for a suitable $c_{o} \in(0,1)$. 
Therefore, if we define $\theta^{\star}:=\max \left\{\theta_{1}, \theta_{2},-1+c\right\}$,

$$
\begin{array}{r}
\left|\left\{u>\theta^{\star}\right\} \cap B_{R}\right|+\left|\left\{\theta_{\star}<u \leqslant \theta^{\star}\right\} \cap B_{R}\right| \\
\quad=\left|\left\{u>\theta_{\star}\right\} \cap B_{R}\right|=V(R) \geqslant c_{o} R^{n}
\end{array}
$$

for large $R$. On the other hand, by Theorem 1.3 of [14], we know that

$$
\mathscr{E}\left(u ; B_{R}\right) \leqslant \bar{C} R^{n-2 s},
$$

for some $\bar{C}>0$, and so

$$
\begin{gathered}
\bar{C} R^{n-2 s} \geqslant \mathscr{E}\left(u, B_{R}\right) \geqslant \int_{\left\{\theta_{\star}<u \leqslant \theta^{\star}\right\} \cap B_{R}} W(u(x)) d x \\
\geqslant \inf _{r \in\left[\theta_{\star}, \theta^{\star}\right]} W(r)\left|\left\{\theta_{\star}<u \leqslant \theta^{\star}\right\} \cap B_{R}\right| .
\end{gathered}
$$

By (14) and (15), we obtain that (4) holds, thus proving Theorem 1

\section{Appendix - The Fractional Sobolev inequality}

For completeness, we provide here an essentially selfcontained and elementary proof of the Sobolev-type inequality used in this paper (in particular, we will not make use of neither interpolations or Besov spaces). For a more comprehensive treatment of fractional Sobolev-type inequalities see $[5$ and references therein.

In this appendix, we will fix $s \in(0,1)$ (in fact, when $s \in[1 / 2,1)$ some of the statements may be strengthened, see [4]). We recall an elementary estimate, for the proof of which see, e.g., the Appendix of [14] and, in particular, Lemma A.1 there:

Lemma 4. Fix $x \in \mathbb{R}^{n}$. Let $E \subset \mathbb{R}^{n}$ be a measurable set with finite measure. Then,

$$
\int_{\mathscr{C} E} \frac{d y}{|x-y|^{n+2 s}} \geqslant c(n, s)|E|^{-2 s / n},
$$

for a suitable constant $c(n, s)>0$.

Now, we make a general observation about a useful summability property:

Lemma 5. Fix $T>1$. Let $N \in \mathbb{Z}$ and

$$
\begin{gathered}
a_{k} \text { be a bounded, nonnegative, decreasing sequence } \\
\text { with } a_{k}=0 \text { for any } k \geqslant N .
\end{gathered}
$$

Then,

$$
\sum_{k \in \mathbb{Z}} a_{k}^{(n-2 s) / n} T^{k} \leqslant C(n, s, T) \sum_{\substack{k \in \mathbb{Z} \\ a_{k} \neq 0}} a_{k+1} a_{k}^{-2 s / n} T^{k},
$$

for a suitable constant $C(n, s, T)>0$, independent of $N$.

Proof. By (16),

$$
\text { both } \sum_{k \in \mathbb{Z}} a_{k}^{(n-2 s) / n} T^{k} \text { and } \sum_{\substack{k \in \mathbb{Z} \\ a_{k} \neq 0}} a_{k+1} a_{k}^{-2 s / n} T^{k} \text { are convergent series. }
$$


Moreover, since $a_{k}$ is nonnegative and decreasing, we have that if $a_{k}=0$, then $a_{k+1}=0$. Accordingly,

$$
\sum_{k \in \mathbb{Z}} a_{k+1}^{(n-2 s) / n} T^{k}=\sum_{\substack{k \in \mathbb{Z} \\ a_{k} \neq 0}} a_{k+1}^{(n-2 s) / n} T^{k} .
$$

Therefore, we use the Hölder inequality with exponents $\alpha:=n / 2 s$ and $\beta:=n /(n-$ $2 s$ ) as follows:

$$
\begin{aligned}
& \frac{1}{T} \sum_{k \in \mathbb{Z}} a_{k}^{(n-2 s) / n} T^{k}=\sum_{k \in \mathbb{Z}} a_{k+1}^{(n-2 s) / n} T^{k} \\
& \quad=\sum_{\substack{k \in \mathbb{Z} \\
a_{k} \neq 0}} a_{k+1}^{(n-2 s) / n} T^{k} \\
& \quad=\sum_{\substack{k \in \mathbb{Z} \\
a_{k} \neq 0}}\left(a_{k}^{2 s /(n \beta)} T^{k / \alpha}\right)\left(a_{k+1}^{1 / \beta} a_{k}^{-2 s /(n \beta)} T^{k / \beta}\right) \\
& \leqslant\left(\sum_{k \in \mathbb{Z}}\left(a_{k}^{2 s /(n \beta)} T^{k / \alpha}\right)^{\alpha}\right)^{1 / \alpha}\left(\sum_{\substack{k \in \mathbb{Z} \\
a_{k} \neq 0}}\left(a_{k+1}^{1 / \beta} a_{k}^{-2 s /(n \beta)} T^{k / \beta}\right)^{\beta}\right)^{1 / \beta} \\
& \leqslant\left(\sum_{k \in \mathbb{Z}} a_{k}^{(n-2 s) / n} T^{k}\right)^{2 s / n}\left(\sum_{\substack{k \in \mathbb{Z} \\
a_{k} \neq 0}} a_{k+1} a_{k}^{-2 s / n} T^{k}\right)^{(n-2 s) / n}
\end{aligned}
$$

So, recalling (17), we obtain the desired result.

We use the above tools to deal with the measure theoretic properties of the level sets of the functions:

Lemma 6. Let

$$
f \in L^{\infty}\left(\mathbb{R}^{n}\right) \text { be compactly supported. }
$$

For any $k \in \mathbb{Z}$ let

$$
a_{k}:=\left|\left\{|f|>2^{k}\right\}\right|
$$

Then,

$$
\int_{\mathbb{R}^{n}} \int_{\mathbb{R}^{n}} \frac{|f(x)-f(y)|^{2}}{|x-y|^{n+2 s}} d x d y \geqslant c(n, s) \sum_{\substack{k \in \mathbb{Z} \\ a_{k} \neq 0}} a_{k+1} a_{k}^{-2 s / n} 2^{2 k},
$$

for a suitable constant $c(n, s)>0$.

Proof. Notice that

$$
|| f(x)|-| f(y)|| \leqslant|f(x)-f(y)|,
$$

and so, by possibly replacing $f$ with $|f|$, we may consider the case in which $f \geqslant 0$.

We define

$$
A_{k}:=\left\{|f|>2^{k}\right\} .
$$

We remark that $A_{k+1} \subseteq A_{k}$, hence

$$
a_{k+1} \leqslant a_{k} .
$$


We define

$$
D_{k}:=A_{k} \backslash A_{k+1}=\left\{2^{k}<f \leqslant 2^{k+1}\right\} \quad \text { and } \quad d_{k}:=\left|D_{k}\right| .
$$

Notice that

$$
d_{k} \text { and } a_{k} \text { are bounded and they become zero when } k \text { is large enough, }
$$

thanks to (18). Also, we observe that the $D_{k}$ 's are disjoint, that

$$
\bigcup_{\substack{\ell \in \mathbb{Z} \\ \ell \leqslant k}} D_{\ell}=\mathscr{C} A_{k+1}
$$

and that

$$
\bigcup_{\substack{\ell \in \mathbb{Z} \\ \ell \geqslant k}} D_{\ell}=A_{k}
$$

As a consequence of (23), we have that

$$
a_{k}=\sum_{\substack{\ell \in \mathbb{Z} \\ \ell \geqslant k}} d_{\ell}
$$

and so

$$
d_{k}=a_{k}-\sum_{\substack{\ell \in \mathbb{Z} \\ \ell \geqslant k+1}} d_{\ell}
$$

We stress that the series in (24) is convergent, due to (21), thus so is the series in (25). Similarly, we can define the convergent series

$$
S:=\sum_{\substack{\ell \in \mathbb{Z} \\ a_{\ell-1} \neq 0}} 2^{2 \ell} a_{\ell-1}^{-2 s / n} d_{\ell}
$$

We notice that $D_{k} \subseteq A_{k} \subseteq A_{k-1}$, hence $a_{i-1}^{-2 s / n} d_{\ell} \leqslant a_{i-1}^{-2 s / n} a_{\ell-1}$. Therefore

$$
\begin{aligned}
\{(i, \ell) & \left.\in \mathbb{Z} \text { s.t. } a_{i-1} \neq 0 \text { and } a_{i-1}^{-2 s / n} d_{\ell} \neq 0\right\} \\
\subseteq & \left.\subseteq(i, \ell) \in \mathbb{Z} \text { s.t. } a_{\ell-1} \neq 0\right\} .
\end{aligned}
$$

We use (27) and (20) in the following computation:

$$
\begin{aligned}
& \sum_{\substack{i \in \mathbb{Z} \\
a_{i} \neq 1 \neq 0}} \sum_{\substack{\ell \in \mathbb{Z} \\
\ell \geqslant i+1}} 2^{2 i} a_{i-1}^{-2 s / n} d_{\ell}=\sum_{\substack{i \in \mathbb{Z} \\
a_{i-1} \neq 0}} \sum_{\substack{\ell \in \mathbb{Z} \\
\ell \geqslant i+1 \\
a_{i-1}^{-2 / n} d_{\ell} \neq 0}} 2^{2 i} a_{i-1}^{-2 s / n} d_{\ell} \\
& \leqslant \sum_{i \in \mathbb{Z}} \sum_{\substack{\ell \in \mathbb{Z} \\
\ell \geqslant i+1 \\
a_{\ell-1} \neq 0}} 2^{2 i} a_{i-1}^{-2 s / n} d_{\ell}=\sum_{\substack{\ell \in \mathbb{Z} \\
a_{\ell-1} \neq 0}} \sum_{\substack{i \in \mathbb{Z} \\
i \leqslant \ell-1}} 2^{2 i} a_{i-1}^{-2 s / n} d_{\ell} \\
& \leqslant \sum_{\substack{\ell \in \mathbb{Z} \\
a_{\ell-1} \neq 0}} \sum_{\substack{i \in \mathbb{Z} \\
i \leqslant \ell-1}} 2^{2 i} a_{\ell-1}^{-2 s / n} d_{\ell}=\sum_{\substack{\ell \in \mathbb{Z} \\
a_{\ell-1} \neq 0}} \sum_{k=0}^{+\infty} 2^{2(\ell-1)} 2^{-2 k} a_{\ell-1}^{-2 s / n} d_{\ell} \\
& \leqslant \sum_{\substack{\ell \in \mathbb{Z} \\
a_{\ell-1} \neq 0}} 2^{2 \ell} a_{\ell-1}^{-2 s / n} d_{\ell}=S .
\end{aligned}
$$


Now, we fix $i \in \mathbb{Z}$ and $x \in D_{i}$ : then, for any $j \in \mathbb{Z}$ with $j \leqslant i-2$ and any $y \in D_{j}$ we have that

$$
|f(x)-f(y)| \geqslant 2^{i}-2^{j+1} \geqslant 2^{i}-2^{i-1}=2^{i-1}
$$

and therefore, recalling (22),

$$
\begin{aligned}
& \sum_{\substack{j \in \mathbb{Z} \\
j \leqslant i-2}} \int_{D_{j}} \frac{|f(x)-f(y)|^{2}}{|x-y|^{n+2 s}} d y \geqslant 2^{2(i-1)} \sum_{\substack{j \in \mathbb{Z} \\
j \leqslant i-2}} \int_{D_{j}} \frac{d y}{|x-y|^{n+2 s}} \\
& =2^{2(i-1)} \int_{\mathscr{C} A_{i-1}} \frac{d y}{|x-y|^{n+2 s}} .
\end{aligned}
$$

This and Lemma 4 imply that, for any $i \in \mathbb{Z}$ and any $x \in D_{i}$, we have that

$$
\sum_{\substack{j \in \mathbb{Z} \\ j \leqslant i-2}} \int_{D_{j}} \frac{|f(x)-f(y)|^{2}}{|x-y|^{n+2 s}} d y \geqslant c_{o} 2^{2 i} a_{i-1}^{-2 s / n}
$$

for a suitable $c_{o}>0$.

As a consequence, for any $i \in \mathbb{Z}$,

$$
\sum_{\substack{j \in \mathbb{Z} \\ j \leqslant i-2}} \int_{D_{i} \times D_{j}} \frac{|f(x)-f(y)|^{2}}{|x-y|^{n+2 s}} d(x, y) \geqslant c_{o} 2^{2 i} a_{i-1}^{-2 s / n} d_{i},
$$

where $d(x, y)$ denotes the volume element for the product Lebesgue measure on $\mathbb{R}^{n} \times$ $\mathbb{R}^{n}$. Therefore, by (25), we conclude that, for any $i \in \mathbb{Z}$,

$$
\sum_{\substack{j \in \mathbb{Z} \\ j \leqslant i-2}} \int_{D_{i} \times D_{j}} \frac{|f(x)-f(y)|^{2}}{|x-y|^{n+2 s}} d(x, y) \geqslant c_{o}\left[2^{2 i} a_{i-1}^{-2 s / n} a_{i}-\sum_{\substack{\ell \in \mathbb{Z} \\ \ell \geqslant i+1}} 2^{2 i} a_{i-1}^{-2 s / n} d_{\ell}\right] .
$$

By (26) and (29), we have that

$$
\sum_{\substack{i \in \mathbb{Z} \\ a_{i}=1 \neq 0}} \sum_{\substack{j \in \mathbb{Z} \\ j \leqslant i-2}} \int_{D_{i} \times D_{j}} \frac{|f(x)-f(y)|^{2}}{|x-y|^{n+2 s}} d(x, y) \geqslant c_{o} S .
$$

Then, using (30), (28) and (31),

$$
\begin{aligned}
& \sum_{\substack{i \in \mathbb{Z} \\
a_{i-1} \neq 0}} \sum_{\substack{j \in \mathbb{Z} \\
j \leqslant i-2}} \int_{D_{i} \times D_{j}} \frac{|f(x)-f(y)|^{2}}{|x-y|^{n+2 s}} d(x, y) \\
\geqslant & c_{o}\left[\sum_{\substack{i \in \mathbb{Z} \\
a_{i-1} \neq 0}} 2^{2 i} a_{i-1}^{-2 s / n} a_{i}-\sum_{\substack{i \in \mathbb{Z} \\
a_{i-1} \neq 0}} \sum_{\substack{\ell \in \mathbb{Z} \\
\ell \geqslant i+1}} 2^{2 i} a_{i-1}^{-2 s / n} d_{\ell}\right] \\
\geqslant & c_{o}\left[\sum_{\substack{i \in \mathbb{Z} \\
a_{i-1} \neq 0}} 2^{2 i} a_{i-1}^{-2 s / n} a_{i}-S\right] \\
\geqslant & c_{O} \sum_{\substack{i \in \mathbb{Z} \\
a_{i-1} \neq 0}} 2^{2 i} a_{i-1}^{-2 s / n} a_{i}-\sum_{\substack{i \in \mathbb{Z} \\
a_{i-1} \neq 0}} \sum_{\substack{j \in \mathbb{Z} \\
j \leqslant i-2}} \int_{D_{i} \times D_{j}} \frac{|f(x)-f(y)|^{2}}{|x-y|^{n+2 s}} d(x, y) .
\end{aligned}
$$


That is, by taking the last term to the left hand side,

$$
2 \sum_{\substack{i \in \mathbb{Z} \\ a_{i} \neq 0}} \sum_{\substack{j \in \mathbb{Z} \\ j \leqslant i-2}} \int_{D_{i} \times D_{j}} \frac{|f(x)-f(y)|^{2}}{|x-y|^{n+2 s}} d(x, y) \geqslant c_{o} \sum_{\substack{i \in \mathbb{Z} \\ a_{i}-1 \neq 0}} 2^{2 i} a_{i-1}^{-2 s / n} a_{i} .
$$

On the other hand, by symmetry,

$$
\begin{aligned}
\int_{\mathbb{R}^{n} \times \mathbb{R}^{n}} & \frac{|f(x)-f(y)|^{2}}{|x-y|^{n+2 s}} d(x, y)=\sum_{i, j \in \mathbb{Z}} \int_{D_{i} \times D_{j}} \frac{|f(x)-f(y)|^{2}}{|x-y|^{n+2 s}} d(x, y) \\
= & 2 \sum_{\substack{i, j \in \mathbb{Z} \\
j \leqslant i}} \int_{D_{i} \times D_{j}} \frac{|f(x)-f(y)|^{2}}{|x-y|^{n+2 s}} d(x, y) \\
\geqslant & 2 \sum_{\substack{i \in \mathbb{Z} \\
a_{i-1} \neq 0}} \sum_{\substack{j \in \mathbb{Z} \\
j \leqslant i-2}} \int_{D_{i} \times D_{j}} \frac{|f(x)-f(y)|^{2}}{|x-y|^{n+2 s}} d(x, y) .
\end{aligned}
$$

Then, the desired result plainly follows from (32) and (33).

With the above estimates, we are now in the position of completing the elementary proof of the Sobolev-type inequality exploited in our paper:

Theorem 7. Let $s \in(0,1)$. Let $f: \mathbb{R}^{n} \rightarrow \mathbb{R}$ be measurable and compactly supported. Then,

$$
\|f\|_{L^{2 n /(n-2 s)}\left(\mathbb{R}^{n}\right)}^{2} \leqslant C(n, s) \int_{\mathbb{R}^{n}} \int_{\mathbb{R}^{n}} \frac{|f(x)-f(y)|^{2}}{|x-y|^{n+2 s}} d x d y,
$$

for a suitable constant $C(n, s)>0$.

Proof. Of course, we may suppose that

$$
\text { the right hand side of (34) is finite, }
$$

otherwise we are done.

We will prove (34) under the additional assumption that

$$
f \in L^{\infty}\left(\mathbb{R}^{n}\right) .
$$

This does not affect the generality of the result, because if (34) holds for bounded functions, then it holds for the function $f_{N}$ obtained by $f$ by cutting at levels $-N$ and $+N$. Then, denoting by $|f|_{N}$ the function obtained by cutting $|f|$ at level $N$, we see that $|f|_{N}=\left|f_{N}\right|$, so we obtain from the Fatou Lemma that

$$
\begin{gathered}
\liminf _{N \rightarrow+\infty}\left\|f_{N}\right\|_{L^{2 n /(n-2 s)}}=\liminf _{N \rightarrow+\infty}\left(\int_{\mathbb{R}^{n}}\left(|f|_{N}\right)^{2 n /(n-2 s)}\right)^{(n-2 s) /(2 n)} \\
\geqslant\left(\int_{\mathbb{R}^{n}}|f|^{2 n /(n-2 s)}\right)^{(n-2 s) /(2 n)}=\|f\|_{L^{2 n /(n-2 s)} .}
\end{gathered}
$$

Also, by (35) and the Dominated Convergence Theorem, we have that

$$
\lim _{N \rightarrow+\infty} \int_{\mathbb{R}^{n}} \int_{\mathbb{R}^{n}} \frac{\left|f_{N}(x)-f_{N}(y)\right|^{2}}{|x-y|^{n+2 s}} d x d y=\int_{\mathbb{R}^{n}} \int_{\mathbb{R}^{n}} \frac{|f(x)-f(y)|^{2}}{|x-y|^{n+2 s}} d x d y .
$$

From (37) and (38), one deduces (34) for $f$ from the one for $f_{N}$, hence we may and do assume (36). 
We have, using the notation in (19),

$$
\begin{aligned}
& \|f\|_{L^{2 n /(n-2 s)}\left(\mathbb{R}^{n}\right)}^{2 n /(n-2 s)}=\sum_{k \in \mathbb{Z}} \int_{A_{k} \backslash A_{k+1}}|f|^{2 n /(n-2 s)}(x) d x \\
& \quad \leqslant \sum_{k \in \mathbb{Z}} \int_{A_{k} \backslash A_{k+1}}\left(2^{k+1}\right)^{2 n /(n-2 s)} d x \leqslant \sum_{k \in \mathbb{Z}} 2^{2(k+1) n /(n-2 s)} a_{k} .
\end{aligned}
$$

That is,

$$
\|f\|_{L^{2 n /(n-2 s)}\left(\mathbb{R}^{n}\right)}^{2} \leqslant 4\left(\sum_{k \in \mathbb{Z}} 2^{2 k n /(n-2 s)} a_{k}\right)^{(n-2 s) / n} .
$$

Thus, since $(n-2 s) / n<1$,

$$
\|f\|_{L^{2 n /(n-2 s)}\left(\mathbb{R}^{n}\right)}^{2} \leqslant 4 \sum_{k \in \mathbb{Z}} 2^{2 k} a_{k}^{(n-2 s) / n} .
$$

This, (36), Lemma 5 (applied with $T:=2^{2}$ ) and Lemma 6 give the claim.

It may be worth to remark that, from Lemma 4 it follows that

$$
\int_{E} \int_{\mathscr{C} E} \frac{d x d y}{|x-y|^{n+2 s}} \geqslant c(n, s)|E|^{(n-2 s) / n}
$$

for all measurable sets $E$ with finite measure.

On the other hand, we see that (34) reduces to (39) when $f=\chi_{E}$, so (39) (and thus Lemma 4) may be seen as a Sobolev-type inequality for sets.

\section{REFERENCES}

[1] G. Alberti, G. Bouchitté and P. Seppecher, Phase transition with the line-tension effect, Arch. Rational Mech. Anal. 144 (1998), no. 1, 1-46.

[2] L. Ambrosio, G. de Philippis, L. Martinazzi, Gamma-convergence of nonlocal perimeter functionals, Preprint, 2010.

[3] G. Bouchitté, Singular perturbations of variational problems arising from a two-phase transition model, Appl. Math. Optim. 21 (1990), no. 3, 289-314.

[4] H. Brezis, How to recognize constant functions. Connections with Sobolev spaces, Uspekhi Mat. Nauk 57 (2002), no. 4(346), 59-74; translation in Russian Math. Surveys 57 (2002), no. 4, 693-708.

[5] H. Brezis and P. Mironescu, Gagliardo-Nirenberg, composition and products in fractional Sobolev spaces, J. Evol. Equ. 1 (2001), no. 4, 387-404.

[6] L. A. Caffarelli and A Córdoba, Uniform convergence of a singular perturbation problem, Comm. Pure Appl. Math. 48 (1995), no. 1, 1-12.

[7] L. A. Caffarelli, J.-M. Roquejoffre and O. Savin, Nonlocal minimal surfaces, Comm. Pure Appl. Math. 63 (2010), no. 9, 1111-1144.

[8] L. A. Caffarelli and E. Valdinoci, Uniform estimates and limiting arguments for nonlocal minimal surfaces, To appear in Calc. Var. Partial Differential Equations.

[9] N. Dirr and E. Orlandi, In preparation.

[10] A. Garroni and G. Palatucci, A singular perturbation result with a fractional norm, in Variational problems in material science, Progress in Nonlinear Differential Equations and Their Applications 68 (2006), Birkhäuser, Basel, 111-126.

[11] M. d. M. González, Gamma convergence of an energy functional related to the fractional Laplacian, Calc. Var. Partial Differential Equations 36 (2009), no. 2, 173-210.

[12] M. E. Gurtin, On a theory of phase transitions with interfacial energy, Arch. Rat. Mech. Anal. 87 (1985), no. 3, 187-212.

[13] O. Savin and E. Valdinoci, $\Gamma$-convergence for nonlocal phase transitions, Preprint, 2010.

[14] O. Savin and E. Valdinoci, Density estimates for a variational model driven by the Gagliardo norm, Preprint, 2010, available on-line at

www.mat.uniroma2.it/ valdinoc/s-density-estimates.pdf 
Ovidiu SAVIN

Mathematics Department, Columbia University,

2990 Broadway, New York, NY 10027, USA.

Email: savin@math.columbia.edu

ENRICO VALDINOCI

Dipartimento di Matematica, Università di Roma Tor Vergata, Via della Ricerca Scientifica 1, 00133 Roma, Italy.

Email: enrico@mat.uniroma3.it 